\title{
Robot-assisted laparoscopic transperitoneal pyeloplasty case report
}

\author{
Facundo Davaro $^{1 \wedge}$, Jacob Roberts ${ }^{2}$, Lindsay Lombardo ${ }^{1}$ \\ ${ }^{1}$ Division of Urology, Department of Surgery, Saint Louis University, St. Louis, MO, USA; ${ }^{2}$ Saint Louis University School of Medicine, St. Louis, \\ MO, USA \\ Correspondence to: Facundo Davaro, MD. Division of Urology, Saint Louis University, 1008 S. Spring, \#1511, St. Louis, MO 63110, USA. \\ Email: Facundo.davarocomas@health.slu.edu.
}

\begin{abstract}
Ureteropelvic junction obstruction (UPJO) can be caused by various anatomic abnormalities impairing drainage of urine from the kidney to the ureter. Patients often develop hydronephrosis and caliectasis which can lead to decreased function and eventually failure of the ipsilateral renal unit. Our patient presented with right sided abdominal pain and associated nausea. Classically, these symptoms are colicky in nature, can progress to emesis, and are often provoked by episodes of diuresis. Diagnosis involves a nuclear medicine renal scan with administration of Lasix to ascertain renal function and presence of obstruction. Indications for intervention are nuanced but T1/2 greater than 20 minutes and decreased function of the affected side less than $40 \%$ are typical. Our patient demonstrated a T1/2 of 23 minutes with preserved renal function. Robotic dismembered pyeloplasty is becoming the standard of care for urologists given its minimally invasive approach, faster recovery time and superior success to endoscopic approach and comparative success to open surgery. This case adds a visual walk-through of a robotic pyeloplasty to the scientific literature and addresses debated perioperative management strategies such as comparison of surgical approaches and length of trans-anastomotic drainage. Post-operative imaging with repeat nuclear medicine renal scan with Lasix administration to confirm resolution of obstruction is typically performed. Our case concluded with patient becoming symptom free and a Lasix renal scan demonstrating no evidence of post-renal obstruction.
\end{abstract}

Keywords: Pyeloplasty; ureteropelvic junction obstruction (UPJO); robotic surgery; case report

Received: 25 January 2021; Accepted: 22 June 2021; Published: 20 October 2022.

doi: 10.21037 /jovs-21-3

View this article at: https://dx.doi.org/10.21037/jovs-21-3

\section{Introduction}

We present a patient with right sided ureteropelvic junction obstruction (UPJO) secondary to an anomalous crossing vessel. He presents with classic symptoms of UPJO, undergoes standard work up confirming the diagnosis, and is subsequently treated with a robotic dismembered pyeloplasty. Our case is unique in that it provides a video walk-through of the robotic procedure and a review of current evidence for certain debated perioperative decisions. In particular, we address the role of minimally invasive surgery in the treatment of UPJO and trans-anastomotic drainage techniques and duration. We present the following article in accordance with the CARE reporting checklist (available at https://jovs.amegroups. com/article/view/10.21037/jovs-21-3/rc).

\section{Case presentation}

A 36-year-old Caucasian male developed intermittent right abdominal pain, constipation and nausea for one year.

$\wedge$ ORCID: 0000-0003-4116-3343. 


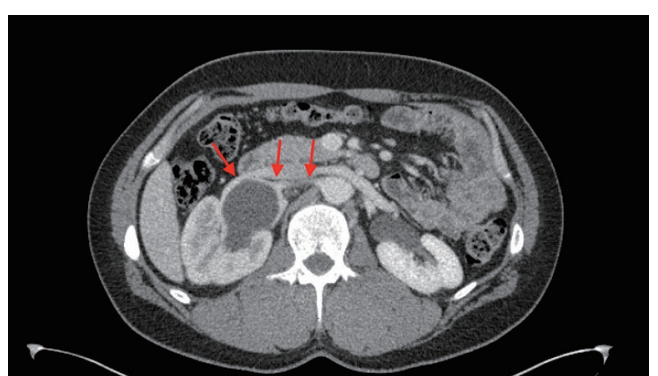

Figure 1 Computed tomography with contrast demonstrating accessory crossing vessels (red arrows) anterior to dilated renal pelvis.

Initial work-up ruled out gastrointestinal disorders such as inflammatory bowel disease. Next, an abdominal ultrasound revealed marked right hydronephrosis. A nuclear medicine renal scan was then performed, demonstrating split function of $54 \%$ on the left and $46 \%$ on the right. A T $1 / 2$ of 23 minutes confirmed right sided obstruction and patient was referred to urology. During office visit, patient corroborated his history, reporting no relevant surgical, genetic or family history. Physical exam was negative, failing to reveal costovertebral angle tenderness, abdominal pain or prior surgical scars. Computed tomography (CT) with contrast and delayed images elucidated the anatomy and established the presence of a crossing vessel (Figure 1). The decision was made to proceed with cystoscopy, right retrograde pyelogram and robotic-assisted laparoscopic pyeloplasty (LP).

Before proceeding with intervention, establishing splitrenal function and determining the cause of the UPJO is paramount. Split-renal function and diagnosing obstruction requires a nuclear medicine scan such as a MAG-3 renal scan (1). Indications for pyeloplasty include $\mathrm{T} 1 / 2$ greater than 20 minutes, $T \frac{1}{2}$ greater than 10 minutes with symptoms indicative of obstruction (i.e., nausea, emesis, ipsilateral flank pain) and/or renal function of less than $40 \%$ on the affected side. Moreover, various nuanced relative indications for treatment exist including chronic pain, recurrent infections, and ipsilateral urolithiasis, among others. The final decision on whether to pursue treatment is based on patient counseling and joint decision making. Similarly, surgical reconstruction is recommended if the obstructed renal unit has greater than $15 \%$ function; meanwhile, less than $15 \%$ function warrants discussion of a nephrectomy (2). However, this is also open for discussion as an asymptomatic patient with an obstructed and poor functioning renal unit does not necessarily require intervention.

Radio-graphical assessment of the cause of the obstruction prior to surgery should also be performed. Various etiologies of UPJO include crossing vessels, high insertion of the ureter, ureteral stricture, and aperistaltic segments are possible. Recommended imaging includes contrast-enhanced CT of the abdomen and/or a retrograde pyelogram in adults, while renal ultrasound is often the preferred imaging modality in children. Regardless of age group, a MAG-3 renal scan with Lasix is a critical aspect of the evaluation of UPJO. It is also important to evaluate the entire ureter ruling out distal strictures, localizing the obstruction which allows for improved surgical planning and port placement. This can best be accomplished with a retrograde pyelogram or even diagnostic ureteroscopy. Endoscopic evaluation can be performed on the day of the pyeloplasty to limit anesthesia events. Endopyelotomy is an appropriate approach for patients as their initial treatment if it measures less than $1 \mathrm{~cm}$ or UPJO is not secondary to a crossing vessel (3). Otherwise, open, laparoscopic or robotassisted pyeloplasty (RAP) is the treatment of choice given the higher success rate. Various pyeloplasty iterations exist including differences in approach, such as trans-peritoneal or retroperitoneal, and technique, such as dismembered, vascular hitch, and pelvic flap pyeloplasty. This list is not exhaustive, but instead, helps illustrate the variety of options available and highlights the importance in surgeon experience and clinical situation in dictating which is the appropriate management.

All procedures performed in this study were in accordance with the ethical standards of the institutional and/or national research committee(s) and with the 2013 Helsinki Declaration. Written informed consent was obtained from the patient.

\section{Preoperative preparation}

Surgery is performed under general anesthesia with endotracheal intubation. First a cystoscopy and retrograde pyelogram is performed with the patient in lithotomy position on a radiolucent bed. A C-arm is used to obtain the pyelogram. The previously obtained abdominal images and the new pyelogram should be available on monitors in the room. The location of the ureteropelvic junction (UPJ) in relation to the umbilicus should be noted for port placement with the camera port at the level of the obstruction.

For our robotic dismembered pyeloplasty, the patient 

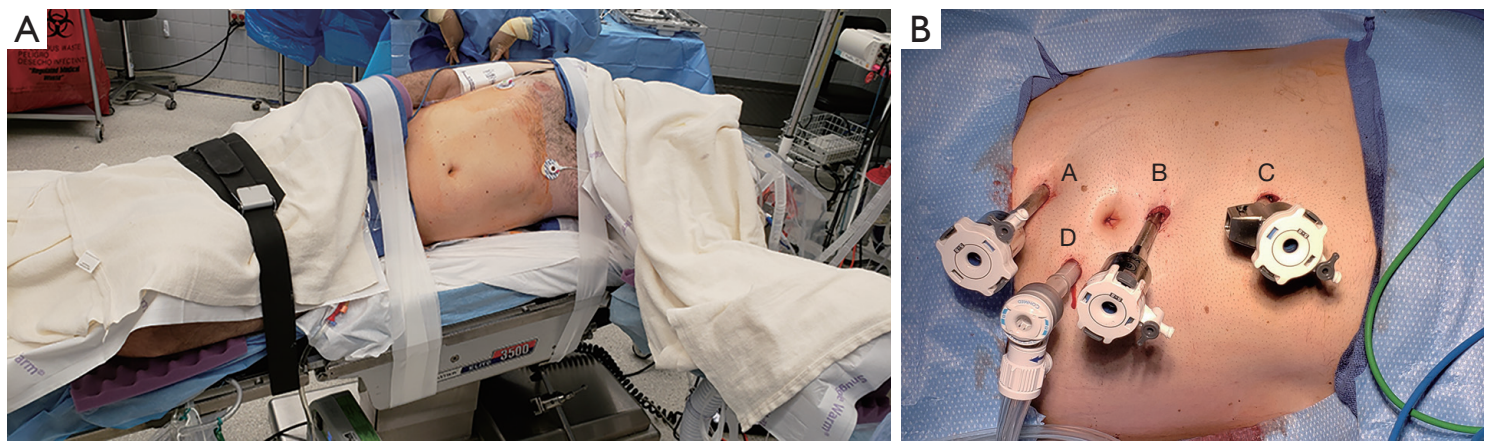

Figure 2 Patient positioning and port placement for right robotic pyeloplasty. (A) Patient positioned in left lateral decubitus position with right arm taped to patient side and left arm extended on arm board. (B) Left-handed robotic port is placed two fingerbreadths superior to anterior sacral iliac spine and lateral to midline (marked A); Robotic camera port is placed just above umbilicus (marked B); right-handed robotic port is placed in the midline one handbreadth cephalad to camera port (marked C); Assistant AirSeal port triangulated between camera and left-handed port (marked D).

is repositioned in the lateral decubitus position with the affected side up. All pressure points are padded. Axillary role should be used. In right sided procedures, the left arm can be placed on an arm board or taped along the side, opposite for left-side procedures (Figure $2 A$ ). If using an arm board, its positioning should allow 90-degree flexion of the shoulder providing adequate room for the robotic arms.

In case of emergency conversion to open, positioning should accommodate a flank incision. Open instruments should be available.

\section{Equipment preferences and cards}

Robotic dismembered pyeloplasty can be done with both da Vinci XI and SI systems (Intuitive Surgical Inc., Sunnyvale, California, USA). This review includes videos using the $\mathrm{Da}$ Vinci XI system. We use robotic ports and an 8-mm AirSeal assistant port providing stable pneumoperitoneum and constant smoke evacuation. We use the Veress technique to achieve pneumoperitoneum. Robotic instruments include fenestrated grasping forceps or Maryland forceps, with bipolar conduction in the non-dominant arm. The scissors with the monopolar conduction are on the dominant arm. A 30-degree camera is used. 4-0 Vicryl sutures are used for the anastomosis in this patient, although barred absorbable suture can also be used per surgeon preference. A double-J stent sized according to patient's height is used. A glide wire and 14-gauge angiocatheter are used to introduce the stent into the peritoneum. A 19-Fr Blake drain is used for drainage.

\section{Procedure}

The anatomic landmarks including the ribs, xyphoid process, umbilicus, and iliac crest are identified. Port sites are marked a hand breadth apart in the midline in a straight line. If using the da Vinci SI, or if the patient has a large space, the left arm port can be placed more lateral creating a triangle. The assistant port is triangulated between the camera and the left arm.

Two finger-breadths below the ribs, the Veress needle is introduced. Pneumoperitoneum is achieved, pressure is set at $15 \mathrm{mmHg}$. Three 8 - $\mathrm{mm}$ robotic ports are placed. An 8 -mm assistant port is placed under direct visualization (Figure 2B). An additional 5-mm assistant port may be required for liver retraction in right sided procedures. The robot is docked behind the patient and the arms are connected. A careful exploration of the abdomen is performed and adhesions are taken down sharply or with cautery.

The right kidney is identified. The white line of Toldt is identified and the colon is reflected off the kidney allowing access to the ureter inferiorly and the renal pelvis superiorly. Kocherization of the duodenum may be necessary depending on the location of the crossing vessel and the hilum. Gerota's fascia is dissected from the renal pelvis. The crossing vessel and ureter are identified. The ureter is mobilized inferiorly away from the gonadal vein. The ureter should not be handled directly with graspers. Cautery should be used sparingly, with care to avoid damaging the ureter and surrounding tissues. Skeletonizing the ureter is avoided to help maintain adequate blood supply. The 


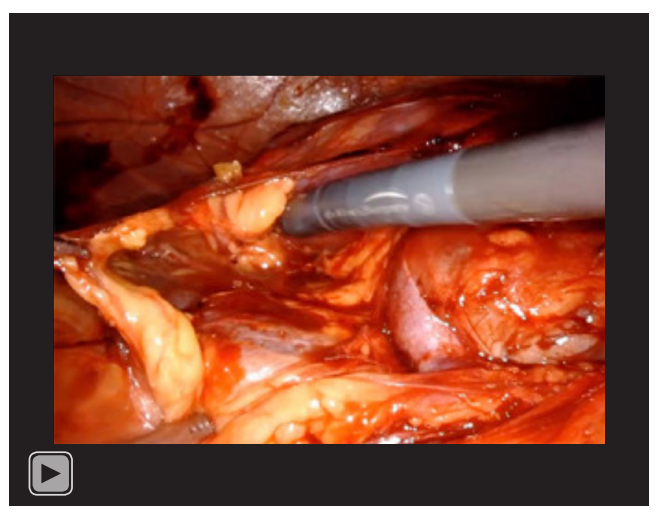

Video 1 The identification of the gonadal vessels and ureter along with the dissection of the anterior crossing vessels.

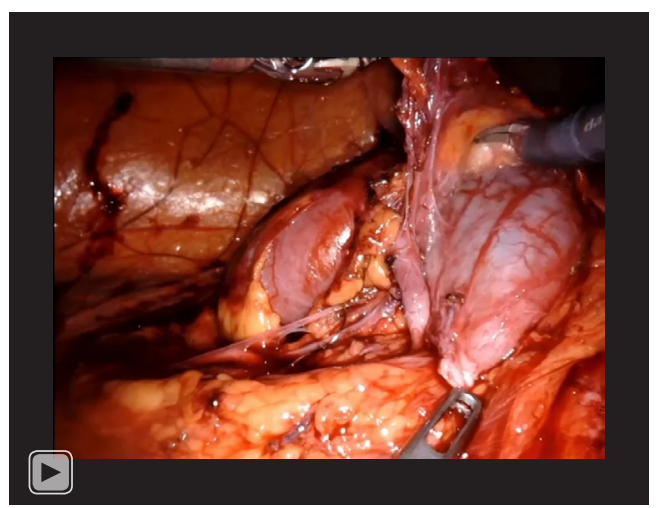

Video 2 Transection and spatulation of the ureter and renal pelvis.

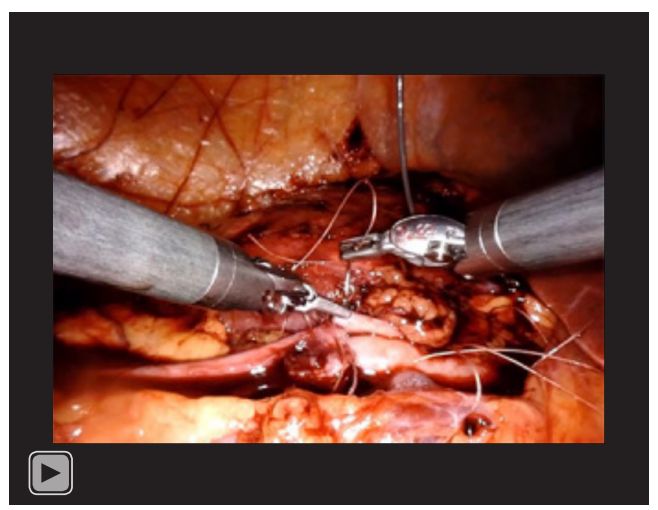

Video 3 Reconstruction of the ureteropelvic junction via a running anastomosis and antegrade stent placement.

dissection is carried up to the crossing vessel at the UPJ and dilated renal pelvis (Video 1).

The crossing vessel is carefully mobilized away from the renal pelvis. A 4-0 Vicryl stay suture is placed in the renal pelvis to aid in handling the renal pelvis and limiting handling of the tissue. This suture should be placed at the apex of the planned incision of the renal pelvis. Suture type is per surgeon preference as long as it is absorbable. The stay suture is passed under the crossing vessel and used to hold the UPJ inferiorly for transection. The UPJ is transected. The renal pelvis stay suture is brought back under the crossing vessel superiorly and further dissected to prepare for anastomosis. The ureter is then brought to the renal pelvis assuring there is adequate length without tension. It is spatulated on the lateral aspect with scissors, for $2 \mathrm{~cm}$ or two lengths of the robotic scissors. Care is taken to avoid grasping the urothelial edges compromising vascular flow. A stay suture may be placed on the ureter if there is minimal peri-ureteral tissue to grasp. The renal pelvis is opened medially with scissors at least $2 \mathrm{~cm}$. Care should be taken to avoid spiraling the incision. Redundant renal pelvis may be excised at this time (Video 2).

A 4-0 vicryl is placed through the apex of the spatulated ureter and at the apex of the renal pelvis incision and tied down. There should be no tension. Two running sutures are placed on either side of the apex suture for the anastomosis. Care is taken to approximate the urothelial edges in a water tight fashion without compromising blood supply. The medial edges are then approximated in the same fashion, but not closed completely, leaving enough room to place the proximal curl of the stent into the renal pelvis. Stent length is based on the patient's height and $2 \mathrm{~cm}$ more than predicted to assure it will reach the bladder. A 14-gauge angio-catheter is passed by the bedside assistant above the anastomosis. The needle is removed, leaving the catheter in place. A wire is passed through the catheter and guided down the ureter. The stent is placed over the wire and the wire removed. The stent is lightly tugged cephalad to confirm the distal curl is in the bladder. If concerned it is not in the bladder, the bladder could be backfilled with fluid through the catheter and should reflux through the stent. Methylene blue can be used to aid in visualization. The proximal curl is placed inside the open renal pelvis. The medial aspect of the anastomosis is approximated with the other running suture. Any excess renal pelvis incision is closed. The sutures are tied to each other (Video 3).

If there is concern for leakage, the bladder can be filled and the anastomosis monitored for leakage. If there is a leak, additional absorbable simple sutures are placed. Hemostasis is achieved. Pneumoperitoneum is reduced to observe any bleeding. A Blake drain is placed away from the anastomosis. 
The colon is placed back over the kidney. Fascia is closed for any port sites $>1 \mathrm{~cm}$. Skin incisions are closed.

\section{Discussion}

Since the first documented pyeloplasty was successfully performed in 1892, the open pyeloplasty (OP) has been the standard of care for the surgical treatment of UPJO (4). With the advancement of laparoscopic and robot-assisted technology, the number of UPJO cases performed via this traditional approach have decreased as these minimally invasive technologies are becoming more ubiquitous. Sukumar et al. investigated trends of UPJO approach over a 10-year span and found a 23 -fold increase in the percentage of minimally invasive surgical approaches for pyeloplasty, from $2.4 \%$ in 1998 to $55.3 \%$ in 2009 (5). As use of the robot for urologic surgery became more prevalent in the 2010's and beyond, it is likely that this percentage will continue to increase (6).

In spite of the advancements in technology and changes in approach, significant differences in surgical success and clinical outcomes have not been borne out in the literature. Rasool et al. conducted a comparison study of 102 patients who underwent pyeloplasty via either OP, LP, or RAP at their institution. Their analysis revealed non-statistically significant differences in success rates, defined as patients who are asymptomatic with normal 6-month nuclear medicine scan or post-operative complications (7). Boylu et al. performed a retrospective review of 42 patients which corroborated these nonsignificant findings (8). The main advantage of OP today lies in its decreased cost and widespread availability. In the pediatric population, Bennett et al. calculated the median cost of OP to be $\$ 10,817$ while RAP was $\$ 11,877(\mathrm{P}=0.03)$ (9). In an era of rising health care costs, the ability to decrease the financial burden without sacrificing successful outcomes is appealing.

As with most comparisons of minimally invasive techniques to open procedures, RAP was shown to decrease other important peri and post-operative metrics. In the same study, Rasool et al. did find significant decreases in peri-operative blood loss, reported level of post-operative pain, and analgesic use in RAP relative to OP. Prolonged operative time is often a critique of robotic surgery. In their analysis, the mean console time was only 104 minutes for RAP; although, when considering docking, port-placement and port closure, the difference in operative times were not clinically significant $(132.06 \pm 30.01$ minutes in OP versus $136.76 \pm 25.10$ minutes in RAP). Importantly, they did show a significant difference in the average hospital length of stay of
$4.12 \pm 1.21$ days after OP versus $2.65 \pm 1.22$ days after RAP (7). The shorter hospital stay was associated with earlier removal of post-operative drain; however, this difference was not statistically significant. These advantages in RAP were supported by Başataç et al. in their review of 56 patients (10). At our institution, it is standard practice to perform all adult pyeloplasties robotically as per surgeon preference. With proper patient education and counseling, either approach is justifiable from a surgical outcomes standpoint.

Post-operative ureteropelvic drainage after pyeloplasty is without much investigation in the adult literature. The standard pyeloplasty procedure often includes placing an indwelling ureteral stent to act as a splint to optimize healing by maintaining alignment of the ureteropelvic anastomosis. A randomized controlled trial of stented versus non-stented OP in 42 pediatric patients demonstrated no differences in surgical outcomes at 3-month nuclear renal scan. As expected, they noted significantly increased levels of post-operative pain and dysuria in the stented cohort which resolved upon stent removal (11). Similar level of evidence is lacking in the adult population. Based on retrospective studies in adults, noting lower success rates and increased rates of urine leak, it is our practice to leave an indwelling stent post-operatively (12). Length of indwelling time is another debated topic, and Danuser et al. investigated this with a prospective study of 100 patients undergoing minimally invasive pyeloplasty. The data revealed that stenting for 1-week was not inferior relative to stenting patients for 4 weeks (13). Our patients typically retain their stents for 2-6 weeks based on patient clinical characteristics and surgeon preference. Successful treatment was confirmed with a post-operative MAG3 renal scan three months after the surgery with a $T 1 / 2$ of 8 minutes and resolution of symptoms.

Patients with symptoms of UPJO, diagnostic imaging confirming obstruction and an anatomic source, such as a crossing vessel, are ideal patients for pyeloplasty. Surgically, it is paramount to ensure a tension free anastomosis via a minimally invasive approach after placing an indwelling ureteral stent for improved healing.

\section{Acknowledgments}

Funding: None.

\section{Footnote}

Reporting Checklist: The authors have completed the CARE 
reporting checklist. Available at https://jovs.amegroups. com/article/view/10.21037/jovs-21-3/rc

Peer Review File: Available at https://jovs.amegroups.com/ article/view/10.21037/jovs-21-3/prf

Conflicts of Interest: All authors have completed the ICMJE uniform disclosure form (available at https://jovs. amegroups.com/article/view/10.21037/jovs-21-3/coif). The authors have no conflicts of interest to declare.

Ethical Statement: The authors are accountable for all aspects of the work in ensuring that questions related to the accuracy or integrity of any part of the work are appropriately investigated and resolved. All procedures performed in this study were in accordance with the ethical standards of the institutional and/or national research committee(s) and with the Helsinki Declaration (as revised in 2013). Written informed consent was obtained from the patient.

Open Access Statement: This is an Open Access article distributed in accordance with the Creative Commons Attribution-NonCommercial-NoDerivs 4.0 International License (CC BY-NC-ND 4.0), which permits the noncommercial replication and distribution of the article with the strict proviso that no changes or edits are made and the original work is properly cited (including links to both the formal publication through the relevant DOI and the license). See: https://creativecommons.org/licenses/by-nc-nd/4.0/.

\section{References}

1. Silay MS, Spinoit AF, Bogaert G, et al. Imaging for Vesicoureteral Reflux and Ureteropelvic Junction Obstruction. Eur Urol Focus 2016;2:130-8.

2. Nakada SY, Best SL. Management of Upper Urinary Tract Obstruction. Wein A, Kavoussi L, Partin A, et

doi: 10.21037 /jovs-21-3

Cite this article as: Davaro F, Roberts J, Lombardo L. Robotassisted laparoscopic transperitoneal pyeloplasty case report. J Vis Surg 2022;8:40. al. Campbell-Walsh Urology. 11th edition. Elsevier, 2015:1104-25.

3. Bergersen A, Thomas R, Lee BR. Robotic Pyeloplasty. J Endourol 2018;32:S68-72.

4. Simforoosh N, Basiri A, Tabibi A, et al. A comparison between laparoscopic and open pyeloplasty in patients with ureteropelvic junction obstruction. Urol J 2004;1:165-9.

5. Sukumar S, Sun M, Karakiewicz PI, et al. National trends and disparities in the use of minimally invasive adult pyeloplasty. J Urol 2012;188:913-8.

6. Intuitive Surgical, Inc. (2018) Annual Report. 2018.

7. Rasool S, Singh M, Jain S, et al. Comparison of open, laparoscopic and robot-assisted pyeloplasty for pelviureteric junction obstruction in adult patients. J Robot Surg 2020;14:325-9.

8. Boylu U, Basatac C, Turan T, et al. Comparison of surgical and functional outcomes of minimally invasive and open pyeloplasty. J Laparoendosc Adv Surg Tech A 2012;22:968-71.

9. Bennett WE Jr, Whittam BM, Szymanski KM, et al. Validated cost comparison of open vs. robotic pyeloplasty in American children's hospitals. J Robot Surg 2017;11:201-6.

10. Başataç C, Boylu U, Önol FF, et al. Comparison of surgical and functional outcomes of open, laparoscopic and robotic pyeloplasty for the treatment of ureteropelvic junction obstruction. Turk J Urol 2014;40:24-30.

11. Nagdeve NG, Bhingare PD, Sarawade P. A Randomized Control Trial Comparing Outcome after Stented and Nonstented Anderson-Hynes Dismembered Pyeloplasty. J Indian Assoc Pediatr Surg 2018;23:186-91.

12. Hopf HL, Bahler CD, Sundaram CP. Long-term Outcomes of Robot-assisted Laparoscopic Pyeloplasty for Ureteropelvic Junction Obstruction. Urology 2016;90:106-10.

13. Danuser H, Germann C, Pelzer N, et al. One- vs 4-week stent placement after laparoscopic and robot-assisted pyeloplasty: results of a prospective randomised singlecentre study. BJU Int 2014;113:931-5. 